\title{
OPEN The impact of pipeline changes and temperature increase in a hospital historically colonised with Legionella
}

\author{
S. Quero ${ }^{1,2,3 凶}$, N. Párraga-Niño ${ }^{1}$, M. Garcia-Núñez ${ }^{1,2,3}$, M. L. Pedro-Botet ${ }^{2,4}$, L. Gavaldà ${ }^{5}$,
} L. Mateu ${ }^{4}$, M. Sabrià ${ }^{2,4} \&$ J. M. Mòdol ${ }^{4}$

Healthcare-related Legionnaires' disease has a devastating impact on high risk patients, with a case fatality rate of 30-50\%. Legionella prevention and control in hospitals is therefore crucial. To control Legionella water colonisation in a hospital setting we evaluated the effect of pipeline improvements and temperature increase, analysing 237 samples over a 2-year period (first year: 129 , second year: 108 ). In the first year, $25.58 \%$ of samples were positive for Legionella and $16.67 \%$ for amoeba. Assessing the distance of the points analysed from the hot water tank, the most distal points presented higher proportion of Legionella colonisation and lower temperatures (nearest points: $6.4 \%$ colonised, and temperature $61.4{ }^{\circ} \mathrm{C}$; most distal points: $50 \%$ and temperature $59.1^{\circ} \mathrm{C}$ ). After the first year, the hot water system was repaired and the temperature stabilised. This led to a dramatic reduction in Legionella colonisation, which was negative in all the samples analysed; however, amoeba colonisation remained stable. This study shows the importance of keeping the temperature stable throughout the circuit, at around $60^{\circ} \mathrm{C}$. Special attention should be paid to the most distal points of the circuit; a fall in temperature at these weak points would favour the colonisation and spread of Legionella, because amoeba (the main Legionella reservoir) are not affected by temperature.

Legionella spp. is the causative agent of Legionnaires' Disease (LD) $)^{1-4}$. Specifically, Legionella pneumophila is associated with the majority of LD cases ${ }^{1-5}$. LD is an atypical pneumonia caused by the inhalation of aerosols containing Legionella. Hospital-acquired LD has been reported to be responsible for up to $14 \%$ of cases of health-care associated pneumonia ${ }^{6,7}$. In 2015 the European Legionnaires' Disease Surveillance Network (ELDSNet) reported that $8 \%$ of LD cases were related to healthcare facilities ${ }^{8}$. The fatality rate of LD is about $10 \%$, but this figure rises to $30-50 \%$ in hospital-acquired LD; therefore, the prevention and control of Legionella in hospitals is essential ${ }^{9}$.

The main reservoirs of Legionella are water-related natural habitats, where it is known to form biofilms in surface interphases or to survive within amoeba. Legionella can colonise man-made water distribution systems, where it multiplies; indeed, these sources have been associated with LD cases and outbreaks ${ }^{10-14}$. In hospitals, cold and hot water distribution systems are the main sources of infection ${ }^{9,15,16}$. The factors that affect Legionella colonisation and growth are the temperature and physicochemical parameters of the water, stagnation, the material used in the pipelines, and the pipelines' age $e^{9,17-19}$. The ability to survive and grow in biofilm and the presence of parasitising amoebas are other important factors in Legionella colonisation.

Prevention and control of Legionella colonisation in health-care units is important because many hospitalised patients have a high risk of Legionella infection. The most effective methods for Legionella disinfection in water distribution systems are chlorination, heating, and copper-silver ionisation ${ }^{3,20}$. According to the World Health Organisation (WHO) guidelines and Spanish (RD865/2003) and Catalan (DOGC352/2004) legislation, hot water systems should be maintained at $50{ }^{\circ} \mathrm{C}$ in the most distal points and at $60^{\circ} \mathrm{C}$ in hot water tanks ${ }^{15,21,22}$. Furthermore, the Catalan regulations stipulate that complementary measures such as superheating or hyperchlorination

${ }^{1}$ Infectious Diseases Unit, Fundació Institut d'Investigació Germans Trias I Pujol, Carretera de Can Ruti, Camí de les Escoles s/n, 08916 Badalona, Barcelona, Spain. ${ }^{2} \mathrm{CIBER}$ de Enfermedades Respiratorias, CIBERES, Av. de Monforte de Lemos, 3-5, 28029 Madrid, Spain. ${ }^{3}$ Institut d'Investigació i Innovació Parc Taulí, I3PT, Parc Taulí, 1, 08208 Sabadell, Barcelona, Spain. ${ }^{4}$ Hospital Universitari Germans Trias i Pujol, Carretera de Canyet, s/n, 08916 Badalona, Barcelona, Spain. ${ }^{5}$ Hospital Universitari de Bellvitge-IDIBELL, Feixa Llarga, s/n., 08907 L'Hospitalet de Llobregat, Barcelona, Spain. ${ }^{\square}$ email: squero@igtp.cat 


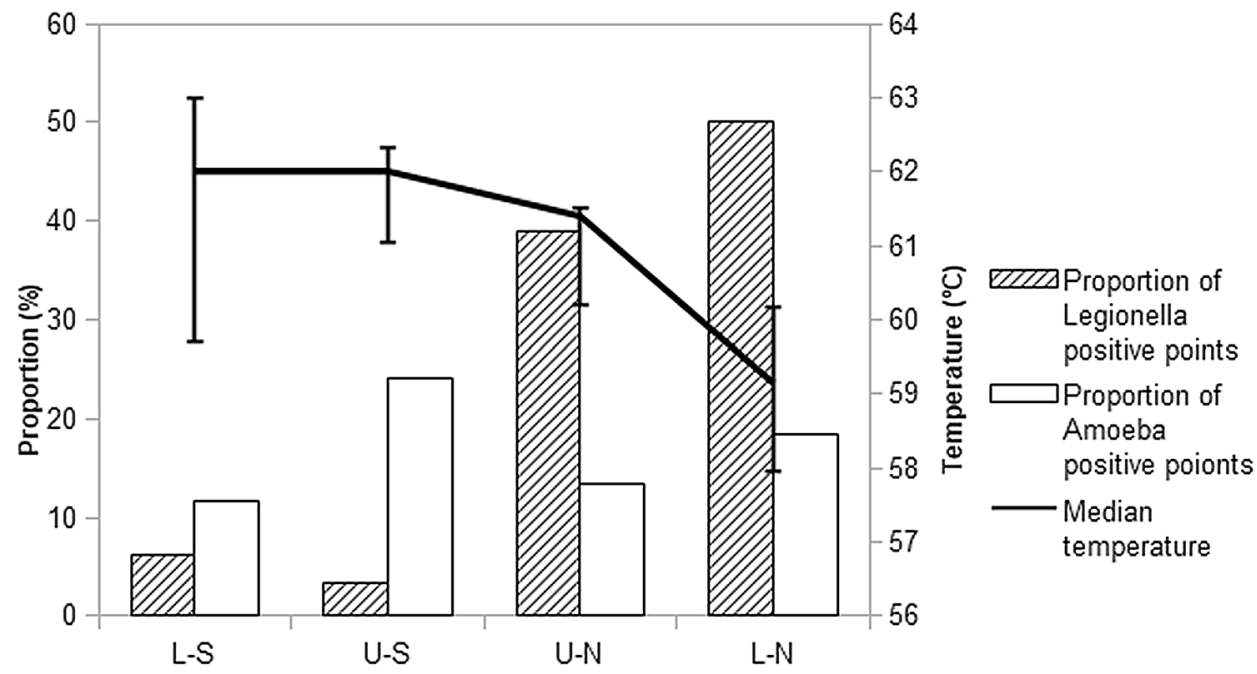

Figure 1. Variations of temperature, Legionella and amoeba colonisation during the observational period (first year) according to the distance from the hot water tank, as defined by the four areas: Lower-South (L-S), UpperSouth (U-S), Upper-North (U-N) and Lower-North (L-N).

flushing should be performed if periodical Legionella control in the water distribution system identifies more than $30 \%$ of points as positive.

The objective of this study was to evaluate the effectiveness of hot water system replacement and temperature increase to prevent Legionella and amoeba colonisation.

\section{Results}

Environmental surveillance. Of the 237 samples collected, 33 were positive for Legionella (13.92\%) and 29 out of 180 samples analysed were positive for amoeba (16.11\%). L. pneumophila sg 2-15 was recovered in all but four positive samples. Two samples were colonised by L. anisa, one by L. pneumophila sg 1 and one by a mix of L. pneumophila sg 1 and Legionella no-pneumophila.

Results according to intervention period-first year (observational period). From 129 samples tested for Legionella presence during the first year, 33 were positive (25.58\%), while 18 out of 108 samples in the amoeba analysis were positive $(16.67 \%)$. The mean temperature was $61.4{ }^{\circ} \mathrm{C}(95 \mathrm{CI} \% 60.19-61.28)$. Sample temperature ranged from 40.7 to $65.5^{\circ} \mathrm{C}$, indicating a high variability in the hot water system.

Temperature level and colonisation. In order to assess the importance of temperature and the presence of Legionella and amoeba, the samples of the first year were classified according to a $60{ }^{\circ} \mathrm{C}$ cut-off. Thirty-seven out of 129 samples were below $60^{\circ} \mathrm{C}(28.68 \%)$ and 31 were positive for Legionella (54.05\%); in contrast, only 13 samples out of $92(14.13 \%)$ above $60^{\circ} \mathrm{C}$ were positive for Legionella $(\mathrm{p}<0.0001)$. Amoeba colonisation was not affected by the sample temperature $\left(19.35 \%\right.$ of samples $<60^{\circ} \mathrm{C}$ and $15.58 \%$ of samples $>60^{\circ} \mathrm{C}$ were colonised by amoeba). There was no correlation between amoeba and Legionella colonisation.

Influence of the distance from the hot water tank and temperature variation. Comparison of the four areas during the first year showed that the further from the hot water tank, the lower the temperature. The mean temperature was $62^{\circ} \mathrm{C}(95 \% \mathrm{CI} 59.7-63)$ in the Lower-South area and $59.15^{\circ} \mathrm{C}(95 \% \mathrm{CI} 57.97-60.16)(\mathrm{p}<0.0001)$ in the Lower-North area (Fig. 1). The water temperature fell in the two areas facing north (South: $62.73{ }^{\circ} \mathrm{C}(95 \% \mathrm{CI}$ 61.9-63.5), North: $58.52^{\circ} \mathrm{C}(95 \%$ CI $\left.58.3-58.8) ; \mathrm{p}<0.0001\right)$. The increase in points colonised by Legionella was associated with the distance from the hot water tank as a result of the fall in temperature (south: $4.92 \%$ Legionella-positive points, north: $44.12 \%$ Legionella-positive points; $\mathrm{p}<0.0001$ ). Amoeba colonisation remained stable throughout the building, and was not affected by the fall in temperature.

Results according to intervention period-second year. After the technical changes, the mean temperature was $62.95^{\circ} \mathrm{C}\left(95 \% \mathrm{CI} 62.7-63.15\right.$, range: $59.8^{\circ} \mathrm{C}$ to $\left.65.9^{\circ} \mathrm{C}\right)$. Only one sample was below $60^{\circ} \mathrm{C}$. Furthermore, the temperature was kept stable in the four areas of the building (Fig. 2). None of the samples were positive for Legionella, although $15.28 \%$ (11/72) were positive for amoeba; there were no differences between the points nearest and furthest away from the hot water tank (Fig. 2).

Clinical surveillance. During the 2-year study period, no cases of hospital-acquired LD were diagnosed. 


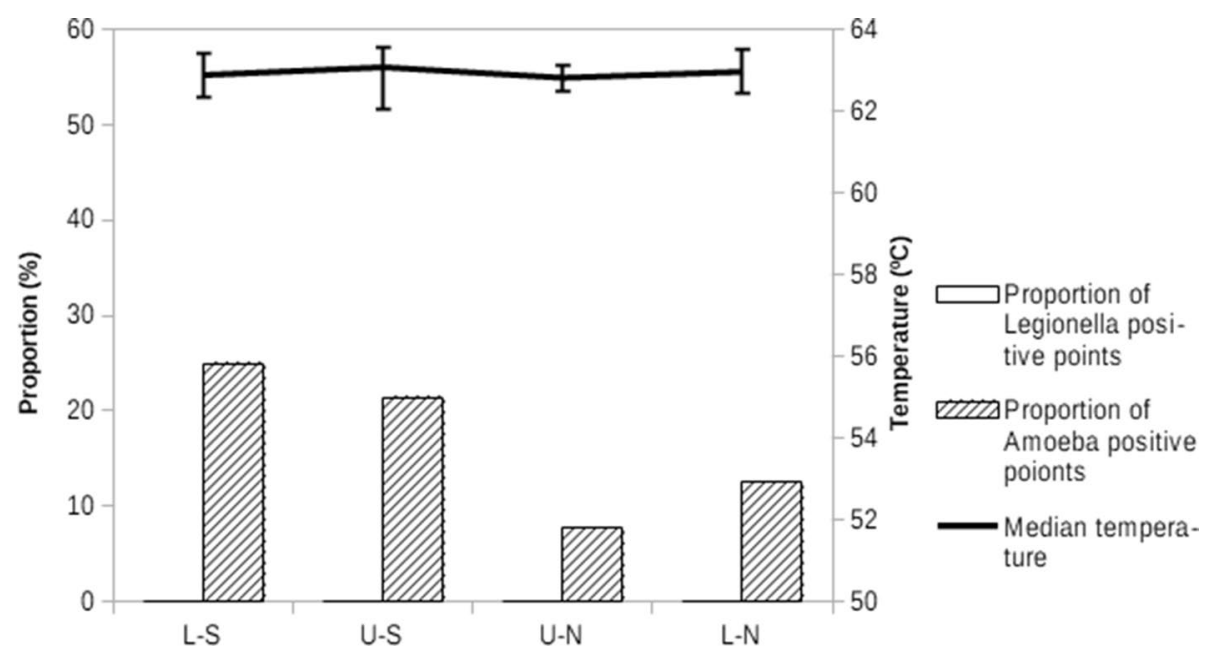

Figure 2. Variations of temperature, Legionella and amoeba colonisation after the implementation of the new measures (temperature increase and change of pipeline material, second year) according to the distance from the hot water tank, defined by the four areas: Lower-South (L-S), Upper-South (U-S), Upper-North (U-N) and Lower-North (L-N).

\section{Discussion}

Large buildings with hot water tank, like hospitals, health-care units and hotels are susceptible to colonisation by Legionella. In these buildings the temperature must be kept high in order to prevent Legionella colonisation and cases of LD. The WHO guidelines and Spanish and Catalan legislation recommend maintaining temperatures of at least $50{ }^{\circ} \mathrm{C}$ in circulating hot water and above $60^{\circ} \mathrm{C}$ in the hot water tank; however, the WHO fact sheet states that at $50^{\circ} \mathrm{C}$ Legionella is able to persist and colonise the pipeline system ${ }^{23}$.

The hospital where this study was performed has a past history of Legionella colonisation. Several disinfection measures were applied, but none were able to completely eradicate the bacterium ${ }^{24,25}$. The intervention described here to eliminate Legionella colonisation showed the importance of the pipeline material and of maintaining the water at a temperature of $60{ }^{\circ} \mathrm{C}$ in the distal points. The points below $60^{\circ} \mathrm{C}$ were the ones that presented the highest rates of colonisation.

Before the replacement, the water temperature in the pipeline in the area nearest the hot water tank was around $60^{\circ} \mathrm{C}$ but the temperature was lower at points further away: most of the samples below $60{ }^{\circ} \mathrm{C}$ were from the Lower-North area, the one furthest away from the tank. The larger a suboptimal pipeline system is, the greater the heat loss. Although the temperature of circulating water started at $60^{\circ} \mathrm{C}$, this level was not maintained across the entire pipeline network, and at the points with lower temperatures Legionella was able to colonise the site and persist inside the circuit.

In the second year, increasing the temperature and changing the pipeline system (i.e., replacing galvanised iron with PVC) achieved a stable temperature throughout the building, with all but one sample remaining above $60^{\circ} \mathrm{C}$. This measure was able to eradicate the Legionella colonisation in the building; however, since amoeba are still present in the pipeline and we did not evaluate the VBNC Legionella, the risk of pipeline Legionella recolonisation remains if the temperature falls once more.

Corrective measures and thermal regimes have been implemented in large buildings to eradicate Legionella from water systems. Gavaldà et al. concluded that a combination of corrective measures and raising the temperature at the end points to $55^{\circ} \mathrm{C}$ might reduce the presence of Legionella ${ }^{26}$. However, after implementing the same measures, Bédard et al. achieved a reduction of Legionella colonisation in only one of the two systems analysed ${ }^{27}$.

Maintaining the temperature of the circulating water above $60^{\circ} \mathrm{C}$ entails a high energy cost. An alternative measure for preventing LD cases in hospital is the installation of point-of-use filters in the faucets. This measure is currently in use in high risk patient areas, but it is expensive and effective only at the installed points; what is more, its effects are only transitory. The installation of point-of-use filters in areas with high risk patients entails a cost of nearly $30000 € /$ year (for 100 points), whereas raising the temperature in the return pipe to the hot water tank from 50 to $55^{\circ} \mathrm{C}$ would suppose an increase of around $32000 € /$ year. So, the cost is similar, but raising the temperature eradicates Legionella throughout the building - not just at the most susceptible points.

One way to reduce the cost of keeping the circulating water above $60{ }^{\circ} \mathrm{C}$ would be to install local heaters in critical areas. This would mean that the temperature in the tank would not have to be $60^{\circ} \mathrm{C}$ in order to keep the circuit at the level required; it would only be necessary to increase the temperature at the "weak" points. Local heaters would drastically bring down the energy costs of heating the tank to high temperatures, and would also avoid overheating of the pipeline, minimise pipeline damage, and reduce the risk of scalding patients.

Local heaters are ideal for buildings with large pipelines and with numerous distal points, such as the one analysed here (with 13 floors and 28 rooms on each floor). These local heaters would also be a good solution for old buildings which use hot water tanks and recirculating water where Legionella can persist in the pipeline, and where colonisation has not been prevented by the technical changes carried out. As the WHO suggested, 
future buildings should be built using a pipeline material that inhibits biofilm formation, and should implement disinfection measures at the beginning of water circulation to prevent Legionella colonisation ${ }^{28}$. Furthermore, temperature control studies should be conducted to identify critical areas where Legionella can grow, and local heaters should be installed to avoid its proliferation.

In the study by Lasheras et al., another factor shown to correlate with Legionella colonisation was water hardness. Those authors concluded that softer water was better in this situation ${ }^{29}$. As our hospital's water is semi-hard ${ }^{30}$, it would be advisable to install a water softener to improve water quality.

Though Legionella was undetectable in the system after the pipeline improvement, amoebas were not affected by this change. Lasheras et al. found a correlation between the presence of amoebas and a larger number of Legionella-positive points, since amoebas are Legionella reservoirs ${ }^{29}$. Although we did not detect Legionella in the second year, we cannot be entirely sure that it was not present in the pipeline; after environmental stresses, Legionella can change its metabolic state to viable but non-culturable (VBNC) and settle in biofilms or parasite amoebas. In any of these states, Legionella is more difficult to detect. In the VBNC state Legionella has low metabolic activity and does not grow in culture media, but in spite of this low growth rate the bacteria retain the features of viable cells such as their integrity and virulence ${ }^{31,32}$.

The impossibility of growing bacteria in VBNC in culture media is a limitation both of our study and of the current regulations regarding environmental Legionella control. The absence of culturable Legionella in water distribution systems does not guarantee water healthiness/safety because VBNC Legionella maintains its capacity to infect and to cause hospital-acquired LD in the most vulnerable/susceptible patients. In this situation there is a clear need to change the current regulations, and to add the detection of VBNC to the culture plate method currently in use. VBNC can be identified by the detection of specific genetic material with PCR using reagents such as propidium monoazide (PMA) or ethidium monoazide bromide (EMA), by antibody detection using flow cytometry protocols, or by reactivation of Legionella cells by adding activating agents or infecting amoeba cells $^{32-34}$. The use of these methods in environmental Legionella control would help to broaden our understanding of Legionella colonisation in water distribution systems. They would also provide information on the power of the disinfectants used in the facility, and on their modes of action.

\section{Conclusions}

In this study we evaluated the colonisation of Legionella and amoeba in a hospital water distribution system, before and after the implementation of two technical changes: the replacement of the pipeline material in circuit 2 , and the increase in the temperature of the hot water tank. A temperature of $55^{\circ} \mathrm{C}$ was achieved at the end of the circuit, just before the water returns to the tank. Moreover, the water temperature in the four areas of the hospital was stable. After the changes, a decrease in Legionella colonisation was observed, while amoeba colonisation remained unaltered. Although the temperature increase is expensive, it improves Legionella control and reduces the likelihood of hospital-acquired $\mathrm{LD}$. Local heaters and water softeners would be a good alternative for reducing the cost of maintaining the temperature required throughout the entire pipeline. These measures would also avoid the use of disposable filters in high-risk-patient areas, reducing both waste and the time needed for their replacement every month. Although Legionella was undetectable after an increase in temperature, efforts are now needed to reduce amoeba colonisation so as to avoid Legionella re-infection. The presence of Legionella in the VBNC state should also be evaluated before water can be considered Legionella-free.

\section{Material and methods}

Setting. The building analysed is a university hospital with 600 beds. This hospital includes renal and bone marrow transplantation programs and houses oncological and haematological wards. The hot water system has two independent water circuits which are fed by a single hot water tank. Circuit 1 supplies the three lowest floors (basement and first and second floors). Circuit 2 supplies floors 3 to 13, which house hospitalisation wards. The water from the hot water tank is distributed to circuit 2 as displayed in Fig. 3. The water from the returning pipes is re-heated and stored in the hot water storage tank, and then redistributed around the building according to requirements. I this study we evaluated the colonisation of circuit 2.

Legionella control program. The disinfection methods used in the hot water system are copper-silver ionisation and heat-and-flush three times yearly. During the study period the levels of copper and silver ions were maintained at $0.2-0.4 \mathrm{ppm}$ and $0.02-0.045 \mathrm{ppm}$ respectively.

For sample analysis, the building was divided into four different areas according to the distance from the hot water tank. The nearest area to the tank was Lower-South (rooms facing south from floors 3 to 8 ), followed by Upper-South (rooms facing south from floors 9 to 13) and Upper-North (floors facing north from floors 9 to 13). Lower-North (rooms facing north from floors 3 to 8 ) was the area furthest from the hot water tank (Fig. 3).

Study design. In order to quantify the effectiveness of the modifications, a before-and-after-changes study was designed. The first year was an observational period prior to the implementation of new measures to eradicate Legionella colonisation. After the observational period, two technical changes were carried out in the pipeline system of circuit 2 to improve Legionella control: the circuit's galvanised iron pipes were replaced by PVC pipes, and the temperature in the hot water tank was raised in order to achieve a temperature of $55^{\circ} \mathrm{C}$ in the return pipe The second-year observation started after the implementation of these technical changes so as to assess their effectiveness.

During the first year, 129 samples were analysed for Legionella and 108 for amoeba and were collected in four rounds (in May, June, September and October). After the modifications of the pipeline system and the 


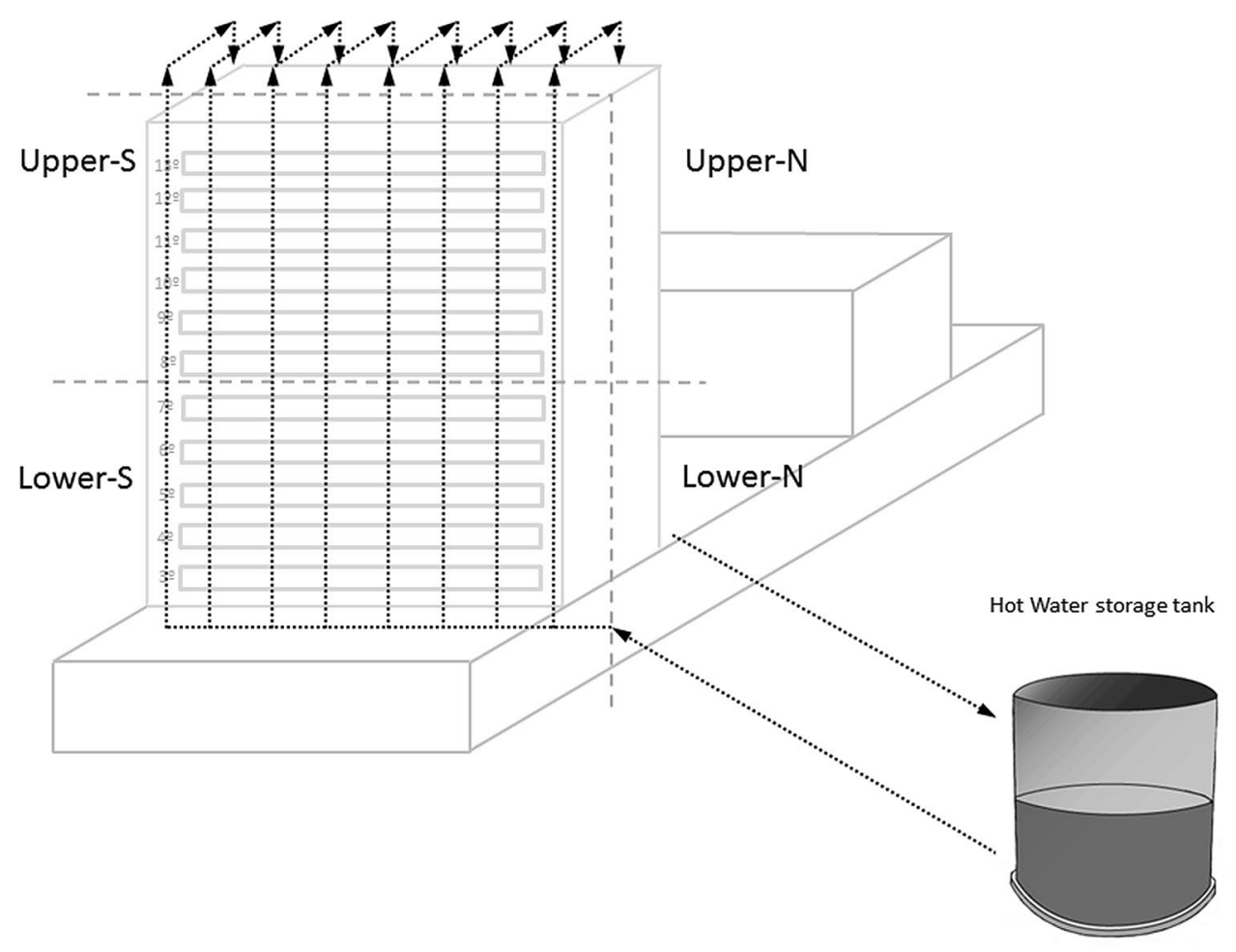

Figure 3. Diagram of hot water circuit 2. The arrows represent the direction of the water. The building is divided into four areas: Lower-S (Lower-South), Upper-S (Upper-South), Upper-N (Upper-North) and Lower-N (Lower-North).

temperature increase, 108 samples were assayed for Legionella over the year and 72 samples for amoeba presence and were collected in three rounds (in May, September and October).

Sampling. Samples from the first year were collected in four rounds, and samples from the second year were collected in three rounds after the pipeline changes.

After 10-30 s of flushing, two litres of water were collected in two sterile polystyrene bottles containing $1 \mathrm{ml}$ of $3 \%$ sodium thiosulfate. Shower and tap heads were dismantled, and internal surfaces were swabbed after the water collection. The swab was then placed into the water sample collected.

The temperature of each sample was measured.

Legionella culture. Legionella detection and quantification was performed in accordance with the ISO protocol 11731:1998(E): Water quality—detection and enumeration of Legionella ${ }^{35}$.

Amoeba culture. A sample of $100 \mathrm{ml}$ of water was filtered through a cellulose nitrate filter, $0.45 \mu \mathrm{m}$ pore size of diameter (Millipore, Bedford, Madison). The filters were inverted on non-nutritive agar (NNA) plates seeded with inactivated Escherichia coli and incubated at $25^{\circ} \mathrm{C}$. The plates were microscopically monitored for outgrowth of amoeba every 3-4 days during 10 days.

Clinical surveillance. Active surveillance of hospital-acquired pneumonia was performed during the study period as described previously ${ }^{36}$. The diagnosis of L. pneumophila pneumonia was based on its isolation from respiratory samples and/or positive results of urinary antigen test. Cases were considered to be hospital-acquired when the patient stayed in the hospital in the 10 days preceding symptom onset.

Data analysis. Descriptive statistics were presented as frequency counts with percentages for categorical variables. Median and 95\% confidence intervals (95\%CI) were calculated for quantitative variables. The comparisons between groups were performed by chi-square test in qualitative/categorical variables and the MannWhitney test in quantitative variables. The differences between groups were defined by $p$-value $<0.05$.

The effect of the temperature was evaluated by establishing a cut-off of $60^{\circ} \mathrm{C}$ in the water sample.

Received: 17 November 2020; Accepted: 1 January 2021

Published online: 21 January 2021 


\section{References}

1. Sopena, N. et al. Prospective study of community-acquired pneumonia of bacterial etiology in adults. Eur. J. Clin. Microbiol. Infect. Dis. Off. Publ. Eur. Soc. Clin. Microbiol. 18, 852-858 (1999).

2. Roig, J., Sabria, M. \& Pedro-Botet, M. L. Legionella spp.: Community acquired and nosocomial infections. Curr. Opin. Infect. Dis. 16, 145-151 (2003).

3. Sabria, M. \& Yu, V. L. Hospital-acquired legionellosis: Solutions for a preventable infection. Lancet Infect. Dis. 2, 368-373 (2002).

4. Sabria, M. et al. A community outbreak of Legionnaires' disease: Evidence of a cooling tower as the source. Clin. Microbiol. Infect. 12, 642-647 (2006).

5. Quero, S. et al. Discriminatory usefulness of pulsed-field gel electrophoresis and sequence-based typing in Legionella outbreaks. Future Microbiol. 11, 757-765 (2016).

6. Barna, Z. et al. Legionella prevalence and risk of legionellosis in Hungarian hospitals. Acta Microbiol. Immunol. Hung. 62, 477-499 (2015).

7. Muder, R. R. et al. Nosocomial Legionnaires' disease uncovered in a prospective pneumonia study. JAMA 249, 3184-3188 (1983).

8. European Centre for Disease Prevention and Control. European Legionnaires' Disease Surveillance Network (ELDSNet). https:// ecdc.europa.eu/en/about-us/partnerships-and-networks/disease-and-laboratory-networks/eldsnet (2018).

9. Borella, P., Bargellini, A., Marchegiano, P., Vecchi, E. \& Marchesi, I. Hospital-acquired Legionella infections: An update on the procedures for controlling environmental contamination. Ann. Ig. Med. Prev. E Comunita 28, 98-108 (2016).

10. Sala, M. R. et al. Community outbreak of Legionnaires disease in Vic-Gurb, Spain in October and November 2005. Euro Surveill. Bull. Eur. Sur Mal. Transm. Eur. Commun. Dis. Bull. 12, 223 (2007).

11. de Olalla, P. G. et al. Community outbreak of pneumonia due to Legionella pneumophila: Importance of monitoring hospital cooling towers. Enfermedades Infecc. Microbiol. Clínica 26, 15-22 (2008).

12. Barrabeig, I. et al. Outbreak of Legionnaires' disease associated with a supermarket mist machine. Epidemiol. Infect. 138, 1823-1828 (2010).

13. Rota, M. et al. Cluster of travel-associated Legionnaires disease in Lazise, Italy, July to August 2011. Euro Surveill. Bull. Eur. Sur. Mal. Transm. Eur. Commun. Dis. Bull. 16, 19982 (2011).

14. Garcia-Nuñez, M. et al. Characterization of unrelated clinical Legionella pneumophila isolates in Catalonia by monoclonal subgrouping and sequence-based typing. Future Microbiol. 11, 865-875 (2016).

15. Lin, Y. E., Stout, J. E. \& Yu, V. L. Controlling Legionella in hospital drinking water: An evidence-based review of disinfection methods. Infect. Control Hosp. Epidemiol. 32, 166-173 (2011).

16. Sabrià, M. et al. Environmental cultures and hospital-acquired Legionnaires' disease: A 5-year prospective study in 20 hospitals in Catalonia, Spain. Infect. Control Hosp. Epidemiol. 25, 1072-1076 (2004).

17. Borella, P. et al. Legionella infection risk from domestic hot water. Emerg. Infect. Dis. 10, 457-464 (2004).

18. Bargellini, A. et al. Parameters predictive of Legionella contamination in hot water systems: Association with trace elements and heterotrophic plate counts. Water Res. 45, 2315-2321 (2011).

19. D’Alessandro, D., Fabiani, M., Cerquetani, F. \& Orsi, G. B. Trend of Legionella colonization in hospital water supply. Ann. Ig. Med. Prev. E Comunita 27, 460-466 (2015).

20. Marchesi, I. et al. Control of Legionella contamination and risk of corrosion in hospital water networks following various disinfection procedures. Appl. Environ. Microbiol. 82, 2959-2965 (2016).

21. DOGC4185, Decret 352/2004. (2017)

22. Real Decreto 865/2003. (2017).

23. World Health Organization. Legionellosis. http://www.who.int/news-room/fact-sheets/detail/legionellosis (2018).

24. Ragull, S. et al. Superheat-and-flush effect on the control of hospital-acquired Legionella infection. Med. Clin. 127, 211-213 (2006).

25. Pedro-Botet Montoya, M. L. et al. The use of molecular epidemiologic markers in the study of an epidemic outbreak of legionnaires' disease of nosocomial origin. Med. Clin. 99, 761-765 (1992).

26. Gavaldà, L., Garcia-Nuñez, M., Quero, S., Gutierrez-Milla, C. \& Sabrià, M. Role of hot water temperature and water system use on Legionella control in a tertiary hospital: An 8-year longitudinal study. Water Res. 149, 460-466 (2019).

27. Bédard, E. et al. Combination of heat shock and enhanced thermal regime to control the growth of a persistent Legionella pneumophila strain. Pathog. Basel Switz. 5, 35 (2016).

28. World Health Organization (WHO). Legionella and the prevention of legionellosis. (2017).

29. Lasheras, A. et al. Influence of amoebae and physical and chemical characteristics of water on presence and proliferation of Legionella species in hospital water systems. Am. J. Infect. Control 34, 520-525 (2006).

30. Aigües de Barcelona. http://www.aiguesdebarcelona.cat/ca/duresa-i-composicio-mineral-de-la-teva-aigua (2018).

31. Kirschner, A. K. T. Determination of viable legionellae in engineered water systems: Do we find what we are looking for?. Water Res. 93, 276-288 (2016).

32. Casini, B. et al. Detection of viable but non-culturable legionella in hospital water network following monochloramine disinfection. J. Hosp. Infect. 98, 46-52 (2018).

33. Taguri, T. et al. A rapid detection method using flow cytometry to monitor the risk of Legionella in bath water. J. Microbiol. Methods 86, 25-32 (2011).

34. Keserue, H.-A., Baumgartner, A., Felleisen, R. \& Egli, T. Rapid detection of total and viable Legionella pneumophila in tap water by immunomagnetic separation, double fluorescent staining and flow cytometry. Microb. Biotechnol. 5, 753-763 (2012).

35. ISO 11731:1998(E). Water quality-detection and enumeration of Legionella.

36. Sopena, N., Sabrià, M. \& Neunos 2000 Study Group. Multicenter study of hospital-acquired pneumonia in non-ICU patients. Chest 127, 213-219 (2005)

\section{Acknowledgements}

The present study was funded by the project PI11/01075, integrated in the National R + D + I and funded by the ISCIII-General Evaluation Branch and the European Regional Development Fund (FEDER) by CIBER de Enfermedades Respiratorias (CIBERES). CIBERES is an initiative of Instituto de Salud Carlos III. The Germans Trias i Pujol Research Institute is included in the CERCA Programme/Generalitat de Catalunya.

\section{Author contributions}

S.Q.: analysed and interpreted the data, wrote the manuscript and approved the final version. N.P.: technical assistant; reviewed the manuscript and approved the final version. M.G.: participated in the study design, implemented the techniques, reviewed the manuscript and approved the final version. M.L.P., L.G., L.M.: reviewed the manuscript and approved the final version. M.S., J.M.M.: designed the study, reviewed the manuscript and approved the final version. 


\section{Competing interests}

The authors declare no competing interests.

\section{Additional information}

Correspondence and requests for materials should be addressed to S.Q.

Reprints and permissions information is available at www.nature.com/reprints.

Publisher's note Springer Nature remains neutral with regard to jurisdictional claims in published maps and institutional affiliations.

(c) (i) Open Access This article is licensed under a Creative Commons Attribution 4.0 International License, which permits use, sharing, adaptation, distribution and reproduction in any medium or format, as long as you give appropriate credit to the original author(s) and the source, provide a link to the Creative Commons licence, and indicate if changes were made. The images or other third party material in this article are included in the article's Creative Commons licence, unless indicated otherwise in a credit line to the material. If material is not included in the article's Creative Commons licence and your intended use is not permitted by statutory regulation or exceeds the permitted use, you will need to obtain permission directly from the copyright holder. To view a copy of this licence, visit http://creativecommons.org/licenses/by/4.0/.

(c) The Author(s) 2021 